\title{
El debate europeo sobre la flexiseguridad: algunas lecciones para el sur
}

\author{
The European debate on flexisecurity: \\ some lessons for the South
}

ÁLVARO VIDAL*

Resumen: El presente artículo analiza el surgimiento y evolución de la estrategia de flexiseguridad en el contexto europeo, sus distintas conceptualizaciones, sus efectos respecto de la función protectora del derecho laboral y las lecciones que se pueden extraer de experiencias previas para Latinoamérica y en particular para el Perú respecto de la subordinación de las políticas socio-laborales a las políticas de generación de empleo.

Palabras clave: flexiseguridad - flexibilidad laboral - protección social - derechos laborales - generación de empleo - políticas socio-laborales

Summary: The present article analyzes the emergence and evolution of flexisecurity strategy in the European context, different conceptualizations, their effects regarding the protective function of labor law and lessons that can be extracted from previous experiences in Latin America and particularly Peru concerning the subordination of social and labor policies on the policies of employment generation.

Keywords: flexisecurity - labor flexibility - social protection - labor rights employment generation - social and labor policies

CONTENIDO: I. INTRODUCCIÓN.- II. EVOLUCION DE LA ESTRATEGIA EUROPEA

DE EMPLEO Y EL ROL DE LA FLEXISEGURIDAD.- II.1. EL "LIBRO VERDE».II.2. COMUNICACIÓN DE LA COMISIÓN: "HACIA LOS PRINCIPIOS COMUNES DELA FLEXISEGURIDAD».-II.3.POLÍTICASCOMUNITARIASDEEMPLEODENTRO DE UNA CONCEPCIÓN DE DERECHOS SOCIALES.- II.3.1 CARTA COMUNITARIA DE DERECHOS SOCIALES FUNDAMENTALES DE LOS TRABAJADORES (ESTRASBURGO, 9 DE DICIEMBRE DE 1989).- II.3.2. CARTA DE DERECHOS FUNDAMENTALES DE LA UNIÓN EUROPEA (NIZA, 7 DE DICIEMBRE DE 2000).II.3.3. ARTICULACIÓN ENTRE POLÍTICA DE EMPLEOY POLÍTICA SOCIAL DE LOS TRATADOS.- II.3.4. ESTRATEGIA EUROPEA DE EMPLEO.- II.3.5. LA NUEVA ESTRATEGIA 2020.- II.3.6. NECESIDAD DE UN MODELO COMUNITARIO DE MÍNIMOS.- III. LOS CAMINOS DE LA FLEXISEGURIDAD.- III.1. LA EVOLUCIÓN DEL CONCEPTO DE FLEXISEGURIDAD.- III.2. ALGUNAS EXPERIENCIAS CONCRETAS DE APLICACIÓN DE LA ESTRATEGIA DE FLEXISEGURIDAD.III.2.1. LA EXPERIENCIA ESPAÑOLA.- III.2.2. LAS REFORMAS EN ITALIA.-

* Profesor de Derecho Laboral y Seguridad Social de la Pontificia Universidad Católica del Perú. Máster en Empleo y Diálogo Social por la Universidad de Castilla-La Mancha. 
III.2.3. LA PERSPECTIVA FRANCESA.- III.2.4. BUSCANDO EN LOS ORÍGENES: LOS CASOS DE DINAMARCA Y PAIISES BAJOS.- IV. EFECTOS EN LA RELACIÓN DE TRABAJO Y RETOS PARA EL DERECHO SOCIAL.- V. ALGUNAS LECCIONES PARA EL SUR.

En la figura que se llama oxímoron, se aplica a una palabra un epíteto que parece contradecirla; así los gnósticos hablaron de luz oscura; los alquimistas, de un sol negro [...] su grosería y su facilidad me tentaron.

JORGE LUIS BORGES, El Zahir

\section{INTRODUCCIÓN}

En medios académicos, empresariales y laborales locales, cada vez con mayor frecuencia, se hace referencia al término flexiseguridad ${ }^{1}$. En algunos casos, con una valoración positiva como modelo que combina lo mejor de la flexibilidad laboral con la seguridad social (básicamente la función de sustitución de ingresos). En otros, de manera más crítica, considerando a la flexiseguridad como medio para emprender reformas de degradación de derechos laborales, trasladando al Estado responsabilidades tradicionalmente empresariales.

Conviene por ello plantearse las siguientes preguntas: icuál es el origen de este término?, ¿cuál fue su evolución?, ¿existe un concepto único?, ¿cuáles son sus límites?, icuáles son sus efectos en la función protectora del derecho del trabajo?, ies replicable este modelo?, y icuáles son las condiciones para que su aplicación sea exitosa?

La reciente reforma laboral española, implementada mediante Real Decreto Ley 3/2012 (10 de febrero de 2012), propone como objetivo la flexiseguridad y en consecuencia apostar por el equilibrio entre la flexibilidad interna y la externa; entre la regulación de la contratación indefinida y la temporal; entre la movilidad interna de la empresa y los mecanismo extintivos de contratación; entre las tutelas que operan en el contrato de trabajo y las que operan en el mercado de trabajo. Equilibrio difícil de alcanzar cuando se analiza el contenido normativo de la reforma y se observa que la balanza se inclina hacia la contratación temporal, la facilitación del despido, la descentralización de la negociación colectiva y las «tutelas del mercado de trabajo», en detrimento de la protección del derecho del trabajo. Todo ello con el objetivo teórico de «facilitar la creación de puestos de trabajo».

1 Si bien el término «flexiguridad» representa una traducción más literal de flexicurity, en el presente trabajo optamos por utilizar el concepto de «flexiseguridad» debido a que manifiesta de manera más evidente la segunda dimensión de este concepto (seguridad) y expresa no solamente las posibles articulaciones de ambos conceptos sino también sus contradicciones. 
Es pertinente recordar que la reforma laboral peruana de inicios de la década de 1990 consideró como su objetivo prioritario generar más puestos de trabajo. La exposición de motivos de la llamada «Ley de Fomento del Empleo» señalaba que «los más recientes desarrollos y evoluciones del pensamiento económico coinciden en asignarle al mercado un rol fundamental en la asignación eficiente de recursos, y el mercado de trabajo tampoco constituye una excepción a esta regla». Argumentaba que gobiernos como los de España, Francia o Italia «se han volcado a dictar medidas que potencien las posibilidades de encuentro entre la oferta y demanda en el mercado laboral, en especial [en] las denominadas franjas débiles del mercado».

Sin embargo, el resultado de la reforma laboral peruana no fue el de generar más empleos sino menos puestos de trabajo y en condiciones más precarias. Como ha sido verificado cuantitativamente por $\mathrm{García}^{2}$, en el año 1991 (cuando se dictó la «Ley de Fomento del Empleo») la tasa de desempleo abierto era de 5,9\% y en los años siguientes se incrementó a 9,4\% (1992), 9,9\% (1993), 8,8\% (1994), 8,2\% (1995) y 7,2\% (1995). Es decir, no existe constatación empírica de que la reforma laboral peruana haya incidido en la generación de más puestos de trabajo. Por otro lado, la precariedad laboral se incrementó: así, tenemos que mientras en el año 1991 el trabajo informal era del 52,7\%, en 1995 fue del 55,1\% y en 2000 del 59,2\%. Es decir, tras la flexibilización laboral no solamente se incrementó el desempleo abierto, sino que además aumentó el trabajo precario.

Por ello, la implementación de políticas de flexibilización laboral (con nuevos o antiguos ropajes) hace necesaria la evaluación comparada de experiencias previas y su impacto real en la economía nacional, el empleo, y el bienestar de los trabajadores. Evaluación que debe hacerse considerando de manera especial las particularidades propias de cada realidad y la pertinencia de aplicar modelos surgidos en contextos económicos, sociales y culturales sustancialmente distintos.

La Estrategia Europea de Empleo (en adelante EEE), principalmente fomentada por la Comisión Europea a partir del año 2006 con la publicación del «Libro Verde» ${ }^{3}$, se ha centrado en gran medida en promover la pertinencia de la flexiseguridad como propuesta eficiente para conciliar las necesidades de generación de empleo y aumento de productividad con una protección flexible del trabajo.

2 GARCíA, Norberto (2005). Competitividad y mercado laboral 1990-2004. Serie macroeconomía del desarrollo. División de desarrollo económico. Santiago de Chile: Comisión Económica para América Latina y el Caribe/Naciones Unidas, 2005. La información señalada es la disponible respecto de los períodos indicados y corresponde a información oficial de Lima metropolitana.

3 «Libro Verde» denominado "Modernizar el Derecho Laboral para afrontar los retos del siglo XXI», presentado por la Comisión de las Comunidades Europeas en el año 2006.

\section{EL DEBATE \\ EUROPEO SOBRE \\ LA FLEXI- \\ SEGURIDAD: \\ ALGUNAS \\ LECCIONES PARA \\ EL SUR}

THE EUROPEAN

DEBATE ON

FLEXISECURITY:

SOME LESSONS

FOR THE SOUTH 
Además de lo contradictorio del término flexiseguridad, aspecto destacado por Umberto Romagnoli ${ }^{4}$, se critica respecto de esta propuesta los siguientes puntos: las dificultades que conllevan implantar este modelo en contextos históricos y culturales distintos de los que le dieron origen; el retroceso en la protección del trabajo y la afectación de la ciudadanía social; el reducido papel otorgado a los sindicatos y lo colectivo; y el traslado de las consecuencias del despido del empleador al Estado y al propio trabajador, lo que, siguiendo a Pérez Rey y Baylos $\mathrm{Grau}^{5}$, podríamos calificar como una socialización de la violencia del poder privado.

Con el objeto de dar respuesta a las interrogantes antes planteadas, a continuación analizaremos el surgimiento y evolución de la estrategia de flexiseguridad en el contexto europeo, sus distintas conceptualizaciones, sus efectos respecto de la función protectora del derecho laboral y las lecciones que se pueden extraer para Latinoamérica y en particular el Perú respecto de la subordinación de las políticas sociolaborales a las políticas de generación de empleo.

\section{EVOLUCIÓN DE LA ESTRATEGIA EUROPEA DE EMPLEO Y EL ROL DE LA FLEXISEGURIDAD}

Desde los tiempos de la fundación de la Unión Europea (UE) con el Tratado de Roma de 1957, esta ha tratado de valorar en un grado u otro la relación entre los objetivos sociales y económicos, pasando por tres etapas distintas ${ }^{6}$. La primera es una etapa de subordinación de las políticas sociales a la realización de los objetivos económicos del mercado único. En la segunda, iniciada a partir del Tratado de Maastricht (1992), se destaca la importancia de las políticas sociales con el objeto de lograr una mayor integración de la ciudadanía europea. En la tercera, introducida por el Tratado de Ámsterdam (1997), se incorpora el título de empleo en el Tratado de las Comunidades Europeas y se implementa el Método Abierto de Coordinación (MAC) para la EEE a través del llamado Proceso de Luxemburgo (1997) y su evolución posterior hasta

4 Umberto Romagnoli ha señalado que al fin y al cabo flexi-security es, en sentido estricto, un oxímoron $y$, como tal, consiste en una pareja de palabras opuestas que expresa una media verdad. La mitad oculta de esta verdad es que solo se puede alcanzar un sistema eficiente de flexi-security mediante la conocida política de dos tiempos, de los cuales, sin embargo, solo se conoce con seguridad el primero porque el segundo no empieza nunca. Sin embargo, este oxímoron no solo es caro, sino también difícil de alcanzar. En ROMAGNOLI, Umberto. «Valoración sobre el Libro Verde». Ponencia presentada en las Jornadas sobre el Libro Verde y el Futuro del Derecho del Trabajo. Noviembre de 2007. Auditori del Palau de la Generalitat de Catalunya.

5 BAYLOS, Antonio \& Joaquín PÉREZ REY. El despido o la violencia del poder privado. Madrid: Trotta, 2009.

6 Ver al respecto LANDA ZAPIRAIN, Juan Pablo. «Introducción». En LANDA ZAPIRAIN, Juan Pablo (coord.). Estudios sobre la estrategia europea de la flexiseguridad: una aproximación crítica. Albacete: Bomarzo, 2009, pp. 11-24. En este punto, el autor sigue a GOETSCHY, Janine. «The implications of the Lisbon Strategy for the future of social Europe: on the road or new age?». The International Journal of Comparative Labour Law and Industrial Relations, XXIII, 4 (2007), pp. 500-501. 
las directrices integradas de la EEE con las Orientaciones Generales de la Política Económica (OGPE) en 2005.

Según Goetschy el debate europeo sobre la flexiseguridad iniciado en 2006 podría suponer una cuarta etapa cuya consideración es más evidente con las nuevas Directrices de la EEE para el período 20082010, en las que se busca conciliar los objetivos sociales y económicos en término iguales. Desde esta perspectiva, la agenda social renovada adoptada por la Comisión en julio de 2008 incluye el objetivo último de la Estrategia de Lisboa: el desarrollo humano.

Juan Pablo Landa opina que, de resultar correcta esta percepción, ella significaría que la UE busca combinar «la seguridad en el empleo y en la renta de las personas con la flexibilidad en el mercado de trabajo, en la organización de la producción y en las relaciones laborales»?

Sin embargo, existen voces sumamente críticas frente al camino seguido en la práctica por la estrategia de flexiseguridad, sobre todo en su aplicación concreta en algunos países de la UE, ya que su ambigüedad ha permitido en algunos casos una relajación de los mecanismos de protección del trabajo, principalmente mediante la flexibilización del despido. Ello es más claro aún si tenemos en cuenta que, como plantea la propia estrategia de la UE, no existe un único camino, «sino tantos como sus estados miembros deseen emprender».

A continuación desarrollamos la evolución de las políticas europeas de empleo y del concepto de flexiseguridad, a partir del «Libro Verde», la Comunicación de la Comisión: «Hacia los Principios Comunes de la Flexiseguridad», las cartas comunitarias que contienen derechos sociales, y las estrategias europeas de empleo, en su versión original y renovada. Asimismo, destacamos el aporte de Pérez Rey sobre la necesidad de contar con un modelo comunitario de mínimos que sea la base del diseño de políticas sociolaborales en el ámbito europeo.

\section{II.1. El «Libro Verde»}

Este documento titulado «Modernizar el Derecho Laboral para afrontar los retos del siglo XXI» presentado por la Comisión de las Comunidades Europeas, tenía como objetivo generar un debate público sobre cómo modernizar el Derecho Laboral para cumplir con el objetivo de la Estrategia de Lisboa: crecer de manera sostenible, con más y mejores empleos. Para ello el documento menciona que los mercados de trabajo europeos deben afrontar el reto de conciliar una mayor flexibilidad con la necesidad de maximizar la seguridad social, así como reducir la

EL DEBATE

EUROPEO SOBRE

LA FLEXI-

SEGURIDAD:

ALGUNAS

LECCIONES PARA

EL SUR

THE EUROPEAN

DEBATE ON

FLEXISECURITY:

SOME LESSONS

FOR THE SOUTH 
segmentación del mercado de trabajo (reducir las diferencias entre los llamados insiders y outsiders).

En este sentido, propugna que el Derecho Laboral debería promover una mayor flexiseguridad que busque lo siguiente:

- Identificar respuestas satisfactorias al desfase entre realidades del mundo laboral y marcos jurídicos y contractuales existentes

- Fomentar la flexibilidad asociada a la seguridad del empleo con independencia de la forma del contrato

- Fomentar el debate sobre la manera en que los tipos de relaciones contractuales podrían favorecer la creación de empleo, facilitando las transiciones en el mercado de trabajo y fomentando el aprendizaje permanente

- Modernizar el Derecho Laboral, en particular que las PYME puedan sufragar los costes administrativos derivados de la legislación

El «Libro Verde» reconoce que las reformas iniciadas desde mediados de los años noventa tuvieron como efecto una «flexibilización en los márgenes», configurando formas de contratación más flexibles (proliferación de contratos temporales, empresas de trabajo temporal, subcontratación y contratos a tiempo parcial), así como una menor protección contra el despido, lo cual ha conducido a una creciente segmentación del mercado de trabajo. Además, plantea que «sistemas de subsidios de desempleo bien diseñados, junto con políticas activas del mercado de trabajo, parecen constituir la mayor garantía frente a los riesgos que presenta el mercado de trabajo» ${ }^{9}$.

En este sentido se plantean como relevantes para la «modernización» del Derecho Laboral reevaluar temas como:

- Una adecuada gestión de las transiciones profesionales (sean involuntarias como el despido y el desempleo o voluntarias como los permisos de formación y las responsabilidades familiares)

- La adopción de medidas para combatir el trabajo oculto como iniciativas de información, mayor control en el cumplimiento de la legislación o aplicación de presunciones legales obligatorias

- La aplicación de igualdad de trato y responsabilidad conjunta y solidaria en el caso de relaciones de trabajo triangulares

- La eficiente ordenación del tiempo de trabajo 
- Medidas de protección de los trabajadores en un contexto de libre circulación a nivel comunitario

Baylos y Pérez Rey ${ }^{10}$ señalan que, independientemente del sesgo ideológico, el «Libro Verde» contiene una serie de temas que requieren un análisis crítico:

- La contratación flexible: trabajo temporal, a tiempo parcial, autónomo y por cuenta propia

- Las «transiciones» en las situaciones de empleo, específicamente las referidas a la tutela de la extinción por causa injustificada mediante el seguro de desempleo y los mecanismos de formación profesional

- La delimitación de las nociones de trabajo oculto, no declarado o irregular, y el trabajo económicamente dependiente aunque prestado bajo régimen de autonomía

- La reconsideración de las relaciones de trabajo triangulares

- La ordenación del tiempo de trabajo como campo de experimentación del trabajo flexible determinado unilateralmente

- La posible definición de una noción comunitaria de trabajador por cuenta ajena como sujeto al que aplicar las normas laborales europeas

- La necesidad de un aparato público que controle los incumplimientos de la legislación laboral y que fuerce su aplicación y vigencia en el espacio europeo (jurisdicción social europea)

Adicionalmente, indican que no se concibe el Derecho Laboral del siglo XXI sin la presencia central del sindicato y de su actuación en la conformación de un marco regulador de las relaciones de trabajo y de empleo. Este marco deberá estar determinado por los derechos humanos y en particular los derechos fundamentales que integran la ciudadanía de la Unión Europea (Carta de Niza).

\section{II.2. Comunicación de la Comisión: «Hacia los principios comunes de la flexiseguridad»}

En el año 2007 la Comisión presentó una comunicación denominada «Hacia los principios comunes de la flexiseguridad: más y mejor empleo 
mediante la flexibilidad y la seguridad $»^{11}$, la cual contenía un conjunto de principios de la flexiseguridad con el objeto de lograr mercados de trabajo más abiertos y con mayor capacidad de respuesta, así como lugares de trabajo más productivos.

Se define a la flexiseguridad como una estrategia integrada para potenciar la flexibilidad y seguridad en el mercado de trabajo, la que puede concebirse y aplicarse a través de cuatro ejes de actuación que pueden reforzarse mutuamente:

- Disposiciones contractuales flexibles y fiables a partir de una legislación laboral, convenios colectivos y una organización del trabajo modernos

- Estrategias globales de aprendizaje permanente que busquen adaptabilidad y empleabilidad continuas

- Políticas activas del mercado laboral eficaces que reduzcan los períodos de desempleo y faciliten las transiciones a nuevos puestos de trabajo

- Sistemas de seguridad social modernos que aseguren una renta adecuada, fomenten el empleo y faciliten la movilidad (cobertura del desempleo, pensiones, prestaciones familiares y salud)

A diferencia del «Libro Verde» se plantea como fundamental la implicación activa de los interlocutores sociales con el objeto de que la flexiseguridad redunde en beneficio de todos. Sus principios comunes podrían ser los siguientes:

- Disposiciones contractuales flexibles, estrategias de aprendizaje permanentes, políticas activas del mercado de trabajo y modernos sistemas de seguridad social

- Equilibrio entre los derechos y las responsabilidades de los empleadores, trabajadores, desempleados y autoridades

- Ausencia de un modelo único y adaptación a las circunstancias, mercados de trabajo y relaciones laborales existentes en cada Estado miembro

- Reducción de la brecha entre los que están dentro del mercado laboral y los que están fuera de él

- Promover la flexibilidad interna como externa, siendo la seguridad social un mecanismo de apoyo de esta y no un desincentivo

11 Comunicación de la Comisión de las Comunidades Europeas al Parlamento Europeo, al Consejo, al Comité Económico y Social Europeo y al Comité de las Regiones. Bruselas 27 de junio de 2007. 
- Promover la igualdad con las mujeres, emigrantes, jóvenes, personas con discapacidad y trabajadores de mayor edad

- Confianza y diálogo entre las autoridades políticas e interlocutores sociales

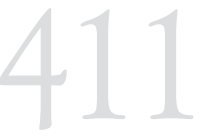

- Políticas presupuestarias sólidas y sostenibles y una distribución justa de los costes y beneficios

Este documento persiste en la flexibilidad externa, incidiendo en la promoción de disposiciones contractuales flexibles y privilegiando la actuación del Estado como responsable de las transiciones laborales mediante sistemas de protección social. Se trasladan los mecanismos de protección del empleo de la relación laboral (responsabilidad del empleador en caso de despido) hacia el Estado (sistemas de protección social).

\section{EL DEBATE \\ EUROPEO SOBRE \\ LA FLEXI- \\ SEGURIDAD: \\ ALGUNAS \\ LECCIONES PARA \\ EL SUR}

THE EUROPEAN

DEBATE ON

FLEXISECURITY:

SOME LESSONS

FOR THE SOUTH

\section{II.3. Políticas comunitarias de empleo dentro de una concepción de derechos sociales}

Como antecedentes del «Libro Verde» y la Comunicación sobre los principios comunes de la flexiseguridad, es necesario destacar la Carta Comunitaria de Derechos Sociales Fundamentales de los Trabajadores (Carta de Estrasburgo) y la Carta de Derechos Fundamentales de la Unión Europea (Carta de Niza), en cuyo marco deben de ser concebidas e implementadas las políticas de empleo en los países miembros.

A continuación, siguiendo a Joaquín Pérez Rey ${ }^{12}$ desarrollamos los principales contenidos de estas cartas y su relación con la EEE para destacar finalmente la importancia de un Modelo Comunitario de Mínimos. Según este autor, supeditar los parámetros sociales a los requerimientos económicos, así como insistir en que el progreso social vendría de la mano de la construcción de un próspero mercado europeo, constituyen el trasfondo ideológico que ha limitado la vertiente social de la integración europea. Por ello, es fundamental retomar la importancia de los instrumentos que reconocen derechos sociales a nivel de la Unión Europea.

\section{II.3.1. Carta Comunitaria de Derechos Sociales Fundamentales de los Trabajadores (Estrasburgo, 9 de diciembre de 1989)}

Los derechos sociales fundamentales reconocidos en esta carta son:

- La libre circulación

- El empleo y la remuneración 
- La mejora de las condiciones de vida y de trabajo

- La protección social

- La libertad de asociación y negociación colectiva

- La formación profesional

- La igualdad de trato entre hombres y mujeres

- La información, consulta y participación de los trabajadores

- La protección de la salud y de la seguridad en el lugar de trabajo

- La protección de los niños y de los adolescentes

- La protección de las personas de edad avanzada y los minusválidos

Esta carta destaca debido a que constituye un instrumento apto para conceder a lo social el protagonismo que requiere en el seno de la UE, aunuqe hay que señalar también su escasa eficacia jurídica.

\section{II.3.2. Carta de Derechos Fundamentales de la Unión Europea (Niza, 7 de diciembre de 2000)}

La importancia de esta carta reside en que luego de la entrada en vigor del Tratado de Lisboa ha asimilado su valor al de los tratados, poniendo fin a la ausencia de una declaración de derechos vinculante en el seno de la UE. Entre los derechos sociales reconocidos en esta carta tenemos los siguientes:

- Prohibición de la esclavitud y del trabajo forzado

- Derecho a trabajar y a ejercer una profesión libremente elegida o aceptada

- Igualdad entre mujeres y hombres en materia de empleo, trabajo y retribución

- Derecho a negociar y celebrar convenios colectivos, en los niveles adecuados, y a emprender, en caso de conflicto de intereses, acciones colectivas para la defensa de sus intereses, incluida la huelga

- Derecho a protección en caso de despido injustificado

- Derecho a trabajar en condiciones que respeten la salud, la seguridad y la dignidad

- Prohibición del trabajo infantil

- Conciliación de la vida familiar y profesional

- Derecho a la seguridad social 


\section{II.3.3. Articulación entre política de empleo y política social de los tratados}

Si bien estas cartas significaron un avance para el reconocimiento de los derechos sociales a nivel comunitario, entre las dificultades que afectan su efectividad destacan el sometimiento de los derechos proclamados al principio de subsidiariedad, así como la imposibilidad de derivar nuevas competencias para las instituciones de la UE.

El Tratado de Funcionamiento de la Unión Europea (TFUE) señala que «la Unión podrá tomar iniciativas para garantizar la coordinación de las políticas sociales de los Estados miembros». Sin embargo, hay aspectos de técnica regulativa que generan dificultades y reflejan cierta subordinación de las políticas sociales al mercado de trabajo. Los aspectos regulatorios que generan dificultades consisten en:

- Desarrollar mediante títulos separados política de empleo y política social, lo cual revela que se ha preferido dejar el empleo a salvo de la política social otorgándole un tratamiento autónomo

- Se ha mantenido el título del empleo en el terreno del soft law, según Miguel Rodríguez-Piñero, en «ausencia de compromisos jurídicos fuertes que los Estados miembros deban asumir, legitimándose a estos a introducir medidas de adaptabilidad y flexibilidad de los mercados de trabajo que no habrían podido adoptar unilateralmente» ${ }^{13}$

Esta opción se sustentaría en la necesidad de «compatibilizar» las políticas de empleo con las orientaciones generales de las políticas económicas de los Estados miembros y de la Comunidad. Sin embargo, se destaca que más allá de la relación de compatibilización o dependencia de las políticas de empleo frente las políticas económicas, los desajustes del mercado de trabajo y principalmente el desempleo se encuentran entre los principales preocupaciones de las políticas comunitarias.

\section{II.3.4. Estrategia Europea de Empleo}

La EEE diseñada como un mecanismo abierto de coordinación (MAC) entre los Estados miembros y la Unión constituiría una muestra más de soft law del que no se puede extraer compromisos vinculantes en un sentido tradicional, por lo que, según Bruno Caurso, constituye un derecho débil de segunda generación ${ }^{14}$.

Esta estrategia recibió un gran impulso en la Cumbre de Lisboa (23 y 24 de marzo de 2000) que planteó como objetivo estratégico de Europa «convertirse en la economía basada en el conocimiento más competitiva y dinámica del mundo, capaz de crecer económicamente de manera

EL DEBATE

EUROPEO SOBRE

LA FLEXI-

SEGURIDAD:

ALGUNAS

LECCIONES PARA

EL SUR

THE EUROPEAN

DEBATE ON

FLEXISECURITY:

SOME LESSONS

FOR THE SOUTH 
sostenible con más y mejores empleos y con mayor cohesión social». Así se planteaba como reto alcanzar el pleno empleo en 2010.

En un primer balance de la estrategia de Lisboa (2005) se verificó la necesidad de «mejorar de manera radical la gobernanza de la estrategia de Lisboa con el fin de hacerla más eficaz y comprensible». Frente a ello se planteó una estrategia simplificada a desarrollarse en ciclos trianuales conforme al esquema previsto en las conclusiones del Consejo Europeo del 22 y 23 de marzo de 2005.

Las Directrices Integradas por el período 2008-2010 (2008/618/CE) dispusieron como objetivos:

- Aplicar políticas de pleno empleo, calidad del trabajo y cohesión social

- Promover un enfoque de trabajo sustentado en el ciclo de vida

- Garantizar mercados de trabajo inclusivos y aumentar el atractivo del trabajo

- Mejorar la adecuación a las necesidades del mercado de trabajo

- Promover flexibilidad combinada con seguridad del empleo.

- Reducir la segmentación del mercado de trabajo, reconociendo el papel de los interlocutores sociales

- Asegurar una evolución de los costes laborales y establecer mecanismos de fijación de salarios que favorezcan el empleo

- Ampliar y mejorar la inversión en capital humano

- Adaptar los sistemas de educación y formación a las nuevas exigencias en materia de competencias

\section{II.3.5. La nueva Estrategia 2020}

En la evaluación realizada por la Comisión Europea de la primera década de la estrategia de Lisboa, si bien se reconoce que las directrices integradas ayudaron a fijar la dirección de las políticas económicas y de empleo nacionales, se señala que su carácter genérico y la falta de jerarquía interna limitaron su impacto en las políticas nacionales. Por ello, se plantean una serie de objetivos cuantificados y directrices más específicas.

La Estrategia 2020 plantea, entre otros, como objetivos cuantitativos para finales de la presente década los siguientes:

- El 75\% de la población entre 20 y 64 años deberá estar empleada

- El 3\% del PIB de la Unión Europea debería ser invertido en Investigación y Desarrollo (I+D) 
- Abandono escolar menor al 10\%

- Al menos 40\% de la generación más joven con estudios superiores

- El riesgo de pobreza debería amenazar a 20 millones de personas menos

La propuesta de directrices más específicas del Consejo (COM 2010 193) contiene las siguientes:

- Aumentar la participación en el mercado laboral y reducir el desempleo estructural, insistiendo en la estrategia de flexiseguridad

- Conseguir una población activa cualificada que responda a las necesidades del mercado laboral. Promover la calidad del trabajo y el aprendizaje permanente

- Mejorar los resultados de los sistemas educativos y de formación

- Promover la inclusión social y luchar contra la pobreza

La Comisión ha planteado una «Agenda para nuevas calificaciones y empleos» consistente en:

- Definir y ejecutar junto con los interlocutores sociales europeos la segunda fase de la flexiseguridad para concretar las mejores formas de gestionar las transiciones económicas

- Adaptar el marco legislativo a las nuevas modalidades de trabajo

- Facilitar y promover la movilidad laboral en el interior de la Unión y adaptar la oferta a la demanda con un apoyo financiero apropiado de los fondos estructurales y promover una política de inmigración prospectiva

- Reforzar la capacidad de los interlocutores sociales, el diálogo social y la cooperación reforzada entre las instituciones del mercado de trabajo

- Aplicar los principios del aprendizaje permanente

- Desarrollar un Marco Europeo de Cualificaciones, Competencias y Ocupaciones como lenguaje común e instrumento operativo

Ante ello, los Estados miembros deberán:

1. Ejecutar planes nacionales de «flexiseguridad» para reducir la segmentación laboral, facilitar las transiciones y conciliar la vida laboral y familiar

2. Revisar la eficiencia de los sistemas impositivos y de beneficios de forma que el trabajo sea atractivo 
3. Promover nuevas formas de equilibrio entre la vida laboral y familiar, así como políticas de envejecimiento activo

4. Promover la aplicación del diálogo social

5. Establecimiento de marcos nacionales de cualificaciones

6. Garantizar competencias y que la formación sea reconocida en toda las etapas

7. Mayor colaboración entre el mundo educativo y el mundo laboral

\section{II.3.6. Necesidad de un modelo comunitario de mínimos}

Para algunos autores, la insistencia en el MAC debería ser replanteada, ya que, si bien ha permitido fomentar el debate sobre las políticas de empleo, hace evidentes los límites de un modelo en el que está ausente la exigibilidad. Como señala Umberto Romagnoli ${ }^{15}$, se trata de una técnica que renunció a perseguir la unificación de los ordenamientos nacionales, proponiéndose más bien aprender a gobernar las diferencias por la fuerza amable de la persuasión y del consenso.

Entre las razones que Terradillos Ormaetxea ${ }^{16}$ considera como causas de la inexistencia de un Derecho Social Europeo (hard law), destacan las siguientes:

- La filosofía liberal originaria de la Unión Europea y la inicial falta de intervención en la política social

- La percepción del Derecho laboral como seña de identidad cultural e histórica de los estados miembros y la reticencia a la armonización

- El déficit de legitimidad de la Unión debido al papel residual del Parlamento Europeo

- El reconocimiento del principio de subsidiariedad (Maastricht, 1992)

- La expansión territorial que ha experimentado Europa, ampliando la brecha cultural, histórica y económica de los estados europeos

- La ampliación de los sujetos implicados en el proceso de formación del Derecho europeo

Siguiendo a Alonso García ${ }^{17}$, se puede señalar que el soft law es un fenómeno que presenta como característica del Derecho restringir la

15 Citado por PÉREZ REY, Joaquín. «Políticas Comunitarias de Empleo».

16 TERRADILlos ORMAETXEA, Edurne. «La modernización del Derecho Laboral en Europa a través del MAC: ¿La emergencia de otro modelo social europeo?». En LANDA ZAPIRAIN, Juan Pablo (coord.). Ob. cit., pp. 59-62.

17 Alonso García, R. «El soft law comunitario». Revista de Administración Pública, 154 (2001), pp. 63-94. Citado por TerRAdillos ORMAETXEA, Edurne. Ob. cit. 
libertad de sus destinatarios pero al que le falta parte de su naturaleza vinculante. Los instrumentos incluidos en esta acepción son muy amplios, como recomendaciones, dictámenes, libros blancos, libros verdes, etcétera, destacando entre sus características su utilidad como:

- Parámetro interpretativo del hard law

- Medio para determinar la aplicación de principios generales del Derecho

- Instrumento para producir efectos jurídicos ad extra, es decir en la esfera jurídica de los Estados miembros y los ciudadanos

- Medio para tener efectos ad intra, es decir influir en el funcionamiento del aparato institucional comunitario mediante códigos de conducta, reglamentos, libros, etcétera.

Según Terradillos, el MAC supone algo más que una simple fórmula de coordinación y se erige como «el método» que engloba a las distintas aplicaciones del soft law; sin embargo, no se ha consolidado como método de resultados ya que las políticas de empleo estatales no han mejorado ni se han aproximado a lo planteado.

Según Pérez Rey, más que dotar a las políticas ocupacionales de elementos vinculantes, sería preciso someter sus procedimientos actuales a los límites derivados de los derechos fundamentales. Las políticas flexibilizadoras que inciden en la reducción de las garantías laborales, como aquellas que operan frente al despido, no se plantean adecuadamente la compatibilidad de estas orientaciones con los derechos fundamentales, concretamente con los reconocidos por la Carta de Niza.

De ello deriva la necesidad de un modelo comunitario de mínimos en materia de relaciones de trabajo y empleo, que identifique los rasgos que deben caracterizar la forma de trabajar en Europa. Su construcción debería fundarse en las cartas de derechos que tienen incidencia en el ámbito comunitario, a saber, la Carta de Niza y la Carta de Derechos Sociales Fundamentales de los Trabajadores de 1989. Una especie de «núcleo central» al que se añadiría las particularidades internas de cada Estado miembro, propiciando una equiparación por la vía del progreso y evitando una competencia fundada en el dumping social.

Así, según señala Monereo Pérez ${ }^{18}$, el bloque normativo del sistema de los tratados puede ser concebido desde una doble perspectiva:

a) En la perspectiva subjetiva, los derechos del empleo como derechos sociales de ciudadanía (derechos individuales).

EL DEBATE

EUROPEO SOBRE

LA FLEXI-

SEGURIDAD:

ALGUNAS

LECCIONES PARA

EL SUR

THE EUROPEAN

DEBATE ON

FLEXISECURITY:

SOME LESSONS

FOR THE SOUTH 
En otras palabras, se entiende al derecho al trabajo como derecho de inclusión social.

b) En la perspectiva objetiva o institucional de las políticas públicas comunitarias del empleo. Es decir, en la ordenación del principio de heteronomía o intervencionismo público en materia de empleo, que implica un conjunto de intervenciones públicas y un aparato institucional.

A partir de lo antes mencionado, la generación de un modelo comunitario de mínimos, derivado de los derechos sociales fundamentales reconocidos en los tratados, debería no solamente reconocer la titularidad de los mismos a nivel europeo (ciudadanía social europea), sino contar con una institucionalidad pública que permita hacerlos efectivos.

\section{LOS CAMINOS DE LA FLEXISEGURIDAD}

La Comunicación de la Comisión «Hacia los Principios comunes de la flexiseguridad», planteó una serie de itinerarios de la flexiseguridad. Sin embargo, los caminos seguidos en la realidad difieren significativamente de los formulados teóricamente. Por ello, consideramos pertinente plantear la evolución teórica del concepto de flexiseguridad y desarrollar algunas experiencias europeas desde una perspectiva crítica.

\section{III.1. La evolución del concepto de flexiseguridad}

Jaspers ${ }^{19}$ señala que la historia del concepto de flexiseguridad no está exenta de ambigüedades. El concepto surgió en la política de empleo del gobierno danés, en un memorando del ministro de Asuntos Sociales y Empleo, Ad Melkert, donde se presentaba su opinión sobre el necesario equilibrio entre flexibilidad y seguridad. Sin embargo, el término flexibilidad fue introducido anteriormente en Holanda en informes sobre la situación del empleo (en la década de los ochenta del siglo pasado).

Ante la constatación de grupos sensibles a las políticas flexibilizadoras (mujeres y minorías étnicas, entre otros), la combinación de flexibilidad y seguridad era presentada como una «redistribución de los derechos a subsidios entre los que tienen y los que no tienen». Es interesante constatar que estas medidas políticas fueron inicialmente dirigidas a restablecer la posición de los grupos débiles, cuyos miembros eran, cada vez más, contratados de manera temporal. 
Antonio Ojeda ${ }^{20}$ a partir de lo desarrollado por la Comisión Europea ${ }^{21}$, diferencia cuatro variantes de la flexibilidad:

a) Flexibilidad externa numérica, referida a la contratación y despido y a la amplitud de uso de contratos temporales

EL DEBATE

b) Flexibilidad interna numérica, sobre la variación de la cantidad de trabajo utilizada en la empresa sin recurrir a contrataciones o despidos; por ejemplo, mediante cambios en la jornada laboral o uso de horas extras o de tiempo parcial

c) Flexibilidad funcional, referida a cambios en la organización del trabajo o a la adaptación a nuevos desafíos como multiárea o rotación de tareas

d) Flexibilidad salarial, sobre el grado de respuesta del coste salarial a las condiciones económicas

Esta clasificación se centra en las vicisitudes y contenidos de la relación laboral. Adicionalmente, como Ojeda señala, debe tenerse en cuenta el impacto de la flexibilidad laboral en los instrumentos de regulación: flexibilidad en las fuentes. Esta se centra principalmente en el menor rol del Estado como regulador (Estado mínimo) y una mayor preponderancia en la función «normativa» de las partes individuales así como de las colectivas. Y, para completar el panorama, no se debe olvidar la flexibilidad en los presupuestos subjetivos, es decir, en el concepto de empleador y trabajador.

Una clasificación similar es la utilizada por Jaspers para desarrollar la «Matriz de combinaciones de flexibilidad y seguridad». En la variable de flexibilidad se pueden diferenciar:

- La flexibilidad numérica que hace referencia a cantidades. La externa está referida a un sistema de colocación y de finalización que permita contratar fácilmente, así como extinguir la relación, haciendo uso de contratos de duración determinada o de empresas de trabajo temporal. La interna se refiere al uso de contratos de duración corta, a tiempo parcial o a distintas horas en distintos días de la semana, o el mes o el año según las necesidades del empresario.

- La flexibilidad funcional está vinculada con medidas cualitativas. La interna está referida a trabajadores polivalentes, organización flexible del trabajo, incluidos la formación para el puesto y el

20 OJEDA AVILÉS, Antonio. La deconstrucción del derecho del trabajo. Madrid: La Ley/ Wolters Kluwer, 2010, pp. 556-558.

21 COMISIÓN EUROPEA. Employment in Europe Report 2006. Luxemburgo: Oficina de Publicaciones Oficiales, 2006, p. 75. Siguiendo a WILTHEGEN, Ton \& otros. «Towards «flexicurity»?: Balancing flexibility and security in EU Member States». Comunicación al XIII Congreso Mundial de la Asociación de Relaciones Industriales. Berlín, 2003.

EUROPEO SOBRE

LA FLEXI-

SEGURIDAD:

ALGUNAS

LECCIONES PARA

EL SUR

THE EUROPEAN

DEBATE ON

FLEXISECURITY: SOME LESSONS FOR THE SOUTH 
salario variable. También la movilidad dentro de la empresa y la formación extraordinaria como alternativa a la terminación de la relación laboral. La externa se manifiesta en la formación fuera del tiempo de trabajo, programas generales de formación, subcontratación o las empresas de trabajo temporal de alta calidad.

Según Altuzarra y Serrano, la flexibilidad salarial consiste en la posibilidad de tener una estructura salarial que pueda responder a las condiciones del mercado y que premie a los trabajadores de acuerdo con su productividad ${ }^{22}$.

Respecto de la seguridad, se define a la seguridad en el trabajo como certeza de permanencia en un empleo determinado con un empresario específico. La seguridad en el empleo o empleabilidad, como la certeza de seguir trabajando aunque no sea con el mismo empleador. La seguridad en los ingresos económicos como protección de los ingresos en caso de cese en el trabajo, bien sea por despido, expedientes de regulación de empleo, enfermedades crónicas, discapacidad o jubilación. Y, finalmente, la seguridad en la compatibilidad entre un trabajo retribuido y las responsabilidades privadas, como el cuidado de niños, familiares ancianos, períodos sabáticos, reducción de horas de trabajo por motivos personales o asistir a programas de formación continua a lo largo de la vida.

El Grupo de Expertos sobre flexiseguridad, en su informe a la Comisión, manifestó que resultaba crucial para el éxito de estas políticas la participación de los interlocutores y el diálogo social.

La matriz propuesta ha sido criticada por Günther Schmid ${ }^{23}$, debido a que los términos flexibilidad y seguridad debían ser usados de una manera más dinámica con el objeto de ofrecer una relación más compleja, ya que no se trata necesariamente un intercambio entre flexibilidad y seguridad. Según este autor la flexibilidad, como ventaja de los empresarios, no significa necesariamente una pérdida de seguridad para los trabajadores. Por ejemplo, la seguridad en el trabajo puede generar una mayor flexibilidad funcional interna sobre la base de la inversión en capital humano y la lealtad entre las partes. La mayor ventaja de la matriz es que permite comparar los distintos sistemas seguidos por cada país y evita aplicar la flexiseguridad como un molde o receta.

Es muy ilustrativo analizar la evolución del concepto de flexiseguridad realizada por Jaspers. Este señala que Wilthagen (uno de los padres académicos del concepto) definió a la flexiseguridad, inicialmente, de 
manera más instrumental, como una «estrategia política que intenta, sincrónicamente y de manera deliberada, reforzar la flexibilidad del mercado de trabajo, la organización del trabajo y las relaciones laborales, por un lado, así como reforzar la seguridad en el empleo además de la seguridad social, especialmente para los grupos más débiles dentro y fuera del mercado de trabajo, por otro lado» ${ }^{24}$.

La crítica realizada a esta definición reside en su estrechez, sustentada en la necesaria sincronización de ambas partes: reforzar la flexibilidad para una (el empresario) y simultáneamente la seguridad para la otra (el trabajador empleado), en tanto se construye pensando principalmente a los grupos más débiles de la fuerza laboral.

Una crítica de fondo al éxito de estas políticas consiste en que la flexiseguridad no se ha alcanzado por una deliberada estrategia política, sino a través de un gradual proceso de luchas políticas y compromisos en los que ha existido un elemento determinante que es el propio desarrollo histórico. Un factor fundamental para su éxito ha sido la cooperación entre el gobierno y los interlocutores sociales, además de una regulación liberal del despido, una alta protección por desempleo y un buen funcionamiento de la política activa del empleo. Tanto en Dinamarca como los Países Bajos la política socioeconómica del gobierno es decidida con, y apoyada por, empresarios y sindicatos.

Según señala Jaspers, Wilthagen, en su definición revisada de flexiseguridad, contextualiza el concepto dentro de la teoría de los mercados de trabajo transicionales. Así, replantea su definición como «un grado de empleo, trabajo, ingresos y una combinación con seguridad que facilite las carreras y currícula de los trabajadores en los mercados de trabajo que estén en una posición débil, y que les permita durar y participar en un mercado de trabajo, junto con inclusión social de alta calidad, al mismo tiempo que permita un grado de flexibilidad numérica (externa como interna), funcional, así como flexibilidad salarial, que facilite a los mercados de trabajo (y a las empresas individuales) un ajuste adecuado y a tiempo a las condiciones cambiantes, a fin de reforzar la competitividad y la productividad $»^{25}$.

Como se ha hecho notar, esta definición se encuadra dentro de los mercados transicionales y los sistemas sociales como la educación, la formación y la protección social (protección frente al desempleo, sistemas de pensiones y cargas familiares).

24 Tomado de KLAMMER, Ute \& Katja TILLMAN (eds.). Flexicurity - Soziale Sicherung und Flexibilisierung der Arbeits- und Lebensverhältnisse. Düsseldorf: WSI/Hans Böcklerstiftung. Citado por JASPERS, Teun. Ob. cit., p. 90.

25 Citado en ibídem, p. 92. 
Luego de considerar la evolución del concepto de flexiseguridad, Jaspers señala que las principales dificultades para su implementación son:

- Las grandes diferencias de estructuras, tradiciones y resultados económicos de los estados miembros, como señaló la Comisión en su Comunicación de 2007

- El dilema no resuelto de si disminuir el nivel de protección de los «insiders» realmente beneficia a los outsiders

- El cuestionamiento (producido por la crisis económica) a las políticas de flexiseguridad y su sostenibilidad, más aun cuando se observa que los trabajadores flexibles están «pagando la factura»

Frente a ello, Jaspers plantea una flexiseguridad decente, donde destaca el ejemplo holandés con una buena conducta del empleador y del empleado y un correcto comportamiento de uno hacia el otro (equilibrio adecuado entre derechos y obligaciones de las partes).

Por otro lado, según señalan Serrano y Altuzarra ${ }^{26}$, debido a que la estrategia propuesta por la Comisión no busca la eliminación de la flexibilidad numérica derivada de la contratación de trabajadores temporales, sino un equilibrio entre esta y la flexibilidad funcional, teniendo en cuenta las nuevas formas de organización del trabajo (trabajo en el centro vs. trabajo en la periferia), es necesario revalorar la función de la negociación colectiva.

Según estos autores, para lograr un verdadero equilibrio entre ambas flexibilidades se debe fortalecer el rol de la negociación colectiva, herramienta fundamental para instrumentalizar la flexibilidad funcional y reducir (o eliminar) el trabajo precario. La búsqueda del trabajo decente permanente tiene, adicionalmente, como fundamento la mayor probabilidad de que una empresa innove y realice actividades de I+D cuando se respeta la «tasa técnica de temporalidad» (umbral en el cual la contratación temporal incide negativamente en la innovación y productividad).

Como se observa el camino de la flexiseguridad debería conducir a privilegiar la flexibilidad funcional, la inversión en I+D y a fortalecer el papel de los interlocutores sociales y la negociación colectiva como mecanismo regulatorio. Un excesivo fomento de la flexibilidad numérica puede conducir a una retracción de la productividad, además de significar una afectación sustancial del derecho al trabajo reconocido a nivel de las cartas comunitarias. 


\section{III.2. Algunas experiencias concretas de aplicación de la estrategia de flexiseguridad}

Jean Michel Servais ${ }^{27}$, sin pretender realizar una clasificación sistemática, ha tratado de identificar varios grupos de países europeos en función de la búsqueda de equilibrio entre la promoción del empleo y la protección social ${ }^{28}$ :

- Primer grupo: compuesto por los Estados del norte de Europa donde el despido es relativamente flexible y el subsidio de desempleo generoso. Estos países buscan una política de reintegración en el empleo.

- Segundo grupo: conformado por el Reino Unido junto a varios países de Europa central y oriental. La relación laboral se extingue con facilidad, la promoción del empleo es activa, pero las prestaciones de desempleo son limitadas.

- Tercer grupo: constituido por pequeños Estados europeos como Austria, Bélgica, Irlanda o Suiza. Buscan el consenso social en la elaboración y puesta en marcha de políticas de empleo y subsidios de desempleo. La eficacia de las mismas varía de un país a otro, pero todos ofrecen facilidades de rescisión del contrato de trabajo.

Esta distinción busca poner de relieve que es muy difícil trasladar modelos de un sistema de un país a otro, debido a que, en todas partes, las instituciones dependen de la historia, del contexto sociopolítico y de la situación económica.

A continuación desarrollamos algunas experiencias europeas con el objeto de evaluar tanto los contenidos desarrollados como el proceso seguido en cada caso, incidiendo en las diferencias y particularidades de cada Estado miembro.

\section{III.2.1. La experiencia española}

Baylos $\mathrm{Grau}^{29}$ ha señaladoque el procesode implantación delaflexibilidad laboral en España se inició tras la promulgación de la Constitución de 1978. Desde entonces se desarrolló una estrategia de culpabilización del derecho del trabajo frente a los procesos de destrucción de empleo. Paralelamente, se escinde el significado político democrático del trabajo

27 SeRVAIS, Jean Michel. «El complicado equilibrio de la promoción del crecimiento, el empleo y la competitividad con la defensa del Estado Social». En LANDA ZAPIRAIN, Juan Pablo (coord.). Ob. cit., pp. 43-45.

28 Para ello, se apoya (entre otros documentos) en SAPIR, André. "Globalisation and the Reform of the European Social Models». Documento base presentado en la reunión del Consejo de Asuntos Económicos y Financieros (ECOFIN) del Consejo de la Unión Europea. Manchester, 2005. Disponible en www.bruegel.org.

29 BaYLos GRAU, Antonio. "Creación de empleo, mercado de trabajo y Derecho del Trabajo en torno a la experiencia española». Relaciones Laborales, 2 (2009), pp. 789-802. Las ideas vertidas en este punto han sido desarrolladas en dicho artículo.

\section{EL DEBATE \\ EUROPEO SOBRE \\ LA FLEXI- \\ SEGURIDAD: \\ ALGUNAS \\ LECCIONES PARA \\ EL SUR}

THE EUROPEAN

DEBATE ON

FLEXISECURITY:

SOME LESSONS

FOR THE SOUTH 
regulado en la Constitución incidiendo principalmente en el rol que las normas jurídico-laborales asumen en la situación del mercado de trabajo. Así, se inició un proceso de implantación de la flexibilidad laboral, fundamentalmente de carácter externo, cuya principal manifestación son las formas temporales de contratación.

Es importante constatar que estas denominadas reformas del mercado de trabajo no tuvieron los resultados esperados. Así, la tasa de desempleo nunca estuvo por debajo del 15\%, llegando en los momentos más álgidos al 22\% de paro. Adicionalmente, se fue construyendo una enorme bolsa de trabajo flexible que llegó al 33\% de los trabajadores asalariados, segmentándose el mercado laboral entre estables y precarios, siendo estos últimos principalmente jóvenes y mujeres.

Adicionalmente, la regulación laboral temporal se confía a la contratación individual, excluyendo al sindicato de su papel protector, con las consabidas consecuencias respecto del desequilibro entre las partes. Este desequilibrio y la ausencia del sindicato y la negociación colectiva se hacen más evidentes entre las trabajadoras mujeres y los jóvenes.

El resultado de la flexibilización fue extremadamente insatisfactorio, no solamente para los trabajadores sino también para las perspectivas de los empresarios de contar con mayor mano de obra calificada y con alta productividad, todo ello en un contexto de desempleo y alta contratación temporal.

Mediante los acuerdos de 1997 se da una «recuperación de la autonomía colectiva y del proyecto sindical como eje de la relación entre derecho del trabajo y su repercusión en el mercado laboral». El eje de esta relación se canaliza ahora a través del contrato para el «fomento del empleo estable». Desde entonces la estabilidad se concibe como un valor y no se le considera un problema.

A partir de 2002, este compromiso se renueva por los sujetos colectivos en cada uno de los acuerdos sobre negociación colectiva que bianual o anualmente se han venido concertando (diálogo social interprofesional), lo cual implica una revalorización del convenio colectivo como instrumento idóneo para la creación de empleo estable.

Pérez Rey, respecto de la versión inicial de la reforma del año 2002, destaca que es un ejemplo de las dificultades que supone el intercambio equilibrado entre flexibilidad y seguridad, ya que la facilitación del despido encontró un aprovechamiento empresarial, cuya repercusión negativa en los trabajadores fue difícil de compensar mediante los sistemas de protección social. 
Todo ello debido a que se traslada al trabajador la responsabilidad del mal funcionamiento del mercado de trabajo y de los servicios públicos de empleo. Esta transferencia de responsabilidad al trabajador se sustentaría en «una emergente ética individualista que presenta al ciudadano como empresario de su propio capital humano, un ser autónomo y responsable de su suerte» ${ }^{30}$.

Además, hay que señalar que la asunción por el Estado de los costes del cese constituye una transferencia de rentas públicas hacia el sector empresarial, el cual resulta beneficiado doblemente por las políticas de facilitación del despido. Esta tendencia ha sido acentuada mediante las modificaciones normativas implementadas durante 2011 y de manera más reciente con la dación del Real Decreto Ley 3/2012 que tiene como objetivo expreso lograr una mayor flexiseguridad.

\section{III.2.2. Las reformas en Italia}

Según Antonio Loffredo ${ }^{31}$ el marco normativo generado después de las reformas ha creado un clara diferenciación de tutelas, sobre todo para quien ha sido contratado después de la mitad de los años noventa, a causa de algunos fenómenos como: a) la fragmentación empresarial y la especialización productiva; b) la regulación de modalidades contractuales cada vez más precarias; c) la formación profesional como política de empleo; d) el aumento de las causas subjetivas en el diseño de nuevos tipos de contratos.

Loffredo destaca que la influencia más evidente de la globalización económica sobre el Derecho del Trabajo ha sido, sin embargo, la pérdida de centralidad del Estado en la disciplina de las relaciones laborales, a la vez que una revalorización de la empresa como un lugar autónomo de creación de reglas y como espacio «virtual» por el desarrollo de su dimensión transnacional.

Efectos de estas tendencias en la empresa son las externalizaciones mediante la liberalización de la cesión de mano de obra y la transmisión de empresas. Mientras que en el trabajador, el resultado es la precariedad laboral, que se manifiesta mediante la nueva regulación de los contratos temporales, contratos a tiempo parcial, contratos de aprendizaje y contratos de inserción. Estos contratos no solamente expresan una mayor flexibilidad externa sino también una mayor flexibilidad en la determinación de las remuneraciones y el tiempo de trabajo.

En el caso de los contratos temporales, el decreto legislativo 368/2001 recurre a la cláusula general que permite la contratación temporal cuando

30 PÉREZ REY, Joaquín. «La modernización del régimen de despido: el debate europeo». En LANDA ZaPIRAIN, Juan Pablo (coord.). Ob. cit., pp. 135-158.

31 LOFFREDO, Antonio. «Profesionalidad y trabajos: los efectos de la reforma italiana del mercado de trabajo». Revista de Derecho Social, 33 (2006), pp. 25 y ss.

EL DEBATE

EUROPEO SOBRE

LA FLEXI-

SEGURIDAD:

ALGUNAS

LECCIONES PARA

EL SUR

THE EUROPEAN

DEBATE ON

FLEXISECURITY:

SOME LESSONS

FOR THE SOUTH 
existan «razones de tipo técnico, productivo, organizativo y sustitutivo». Además, permite el recurso a modalidades contractuales por causas subjetivas, lo que busca incentivar la contratación de trabajadores con mayores dificultades en el mercado, otorgando menores derechos y retribuciones más bajas.

Por ello Loffredo señala que se requiere reconocer un conjunto de derechos como parte del estatus de trabajador, o más precisamente de ciudadano, en lugar de establecer diferencias en su ejercicio, con base en la tipología contractual o la profesionalidad del trabajador. Al punto que la creación de una gran variedad de tipos contractuales, funcionales a los intereses empresariales, de acuerdo a Loffredo «está produciendo una multitud de figuras sociales, forjando sus comportamientos privados, las expectativas de vida y la propia percepción social».

Como se observa, el camino de la flexiseguridad en Italia parece claramente más inclinado hacia la flexibilidad que a la generación de mecanismos de protección, habiendo producido una situación de precariedad laboral en amplios sectores del mercado de trabajo.

\section{III.2.3. La perspectiva francesa}

Antoine Jeammaud ha desarrollado una interesante comparación entre la concepción del mercado de trabajo desde una perspectiva clásica y una heterodoxa, y su impacto en el diseño de políticas laborales. Así, señala que «el mercado de trabajo aparece como el lugar abstracto y el mecanismo, por una parte de la formación del precio del trabajo, y por otra parte del equilibrio entre oferta de trabajo y demanda de trabajo ${ }^{32}$.

Si una ideología liberal clásica opone regla y mercado, los economistas heterodoxos establecen que no podría existir el mercado sin un cierto número de normas y de convenciones (principalmente implícitas).

Los mercados de trabajo reales no han ilustrado jamás el modelo de mercado de trabajo perfecto (perfectamente competencial, dando lugar a transacciones perfectamente libres entre agentes perfectamente inteligentes y racionales bajo el modelo del homo economicus). Las imperfecciones que lo alejan de este modelo teórico se deben a la singularidad de la mercancía ofertada y demandada.

Así, destaca que en el caso francés, el mercado de trabajo ha sido obra de las reglas de derecho, inclusive antes de la existencia del Derecho del Trabajo, mediante normas constitucionales o de Derecho Civil.

Con la regulación propia del Derecho Laboral, el contrato de trabajo, vía el conjunto de reglas legales y convencionales que lo regulan, busca 
procurar un empleo que es más que un puesto de trabajo en el seno de una organización empresarial: constituye un medio de inserción social e incluso de ciudadanía.

El informe Supiot, encargado por la Comisión Europea a un grupo de expertos y entregado en 1999, según señala Jeammaud, proponía hacer de la libertad de trabajo finalmente una libertad concreta. Ello mediante la adaptación de «la otra flexibilidad», aquella que abre paso a las preferencias de los propios trabajadores, gracias a la edificación de un sistema de «derechos sociales de giro». Una especie de Súper Seguridad Social que administra un gran fondo financiado por múltiples fuentes, permitiendo a cada ciudadano alternar libremente, y sin pérdida de renta, actividad asalariada, actividad profesional independiente, formación, participación en la educación de los hijos, cuidado de familiares dependientes, dedicación a actividades sociales, entre otras.

Respecto del «Libro Verde», Jeammaud destaca que sorprende por su aparente ignorancia de las evoluciones ocurridas en los ordenamientos jurídicos de los estados miembros desde hace un cuarto de siglo, así como por la debilidad de sus bases teóricas.

Asimismo, señala que la doctrina de los mercados de trabajo transitorios sustenta el desarrollo de reformas que busquen compatibilizar la creación de un gran sistema redistributivo de rentas respetando al máximo la libertad individual de elección.

Se trataría, pues, de acondicionar las transiciones en cinco espacios: transiciones en el espacio del empleo, entre formación y empleo, entre desempleo y empleo, entre actividades no comerciales y empleo, entre empleo e inactividad. Este modelo derivado de la sistematización de una serie de experiencias nacionales parciales se aproximaría al concepto de ciudadanía social buscando garantizar «la libertad profesional de las personas» del Informe Supiot.

Respecto del efecto de la «modernización» de la protección social en los sistemas de pensiones en Francia, Philippe Martin ha destacado que, luego de las reformas, el nivel de la pensión es determinado por la totalidad de la carrera profesional. Los parámetros que determinan su cuantía son la duración del período de cotizaciones y el nivel de salario percibido. Esta mayor vinculación entre cotizaciones y prestaciones hace menos redistributivo el sistema, lo que afecta a las personas empleadas de manera precaria, «cuyos derechos matemáticamente se aminoran ${ }^{33}$.

EL DEBATE

EUROPEO SOBRE

LA FLEXI-

SEGURIDAD:

ALGUNAS

LECCIONES PARA

EL SUR

THE EUROPEAN

DEBATE ON

FLEXISECURITY:

SOME LESSONS

FOR THE SOUTH 


\section{III.2.4. Buscando en los orígenes: Ios casos de Dinamarca y Países Bajos}

Teun Jaspers ${ }^{34}$ ha destacado que en algunos países nórdicos y los Países Bajos la flexibilización encaja en el deseo de las personas de disponer de condiciones de trabajo flexibles para conciliar trabajo y cuidados, o mantener cierta libertad frente a los calendarios u horarios rígidos de trabajo.

Asimismo, se vincula al deseo de realizar transiciones exitosas durante el ciclo de vida personal, para lo cual se requiere de cualificaciones que permitan progresar en la vida laboral encontrando nuevos empleos. Y, para ello, se plantea la existencia de subsidios de desempleo que faciliten las transiciones y oportunidades de formación. Sin embargo, las políticas de flexiseguridad deben procurar la atracción y retención de la gente en el mercado de trabajo, más que subsidiar la inactividad.

Dinamarca es considerada un ejemplo exitoso de flexiseguridad (ejemplo de buena práctica). El triángulo dorado (golden triangle), considerado la base de este modelo, consiste en (i) proveer a los empresarios de la flexibilidad óptima en la regulación del Derecho del Trabajo (libertad para contratar y despedir); (ii) una mayor seguridad social para los empleados (principalmente subsidios generosos de desempleo); y (iii) una política activa de reingreso en el trabajo (formación y cooperación para el retorno al trabajo).

La ley holandesa de flexibilidad y seguridad que fue presentada al Parlamento en 1997, junto con una ley sobre empresas de trabajo temporal que buscaba una desrregulación en este sector, fue aprobada en 1998 y entró en vigencia en 1999.

En contraprestación por la liberalización de la actividad de las empresas de trabajo temporal, se introdujeron una serie de presunciones sobre la existencia de una relación laboral (en función de la duración del servicio y el tiempo de trabajo), el número de horas trabajadas y la determinación del tipo de contrato. Estas presunciones fortalecieron la posición de los trabajadores en situación de precariedad.

Así, la referida norma holandesa, conocida como «Flex-Act», tiene como principales medidas:

- La liberalización del mercado de trabajo temporal, consistente en la eliminación del permiso para desarrollar actividades y del límite temporal de seis meses para contratar con estas empresas.

- Permitir la cadena de contratos temporales sucesivos. Se permite contratar tres veces consecutivas hasta un máximo de tres años. 
- Las presunciones legales antes mencionadas sobre la existencia de relación laboral.

- La aplicación de una normativa mínima para los trabajadores discontinuos cuando trabajan un limitado número de horas semanales.

- Medidas de conciliación de la vida laboral y familiar, mediante el ajuste del tiempo de trabajo a la solicitud del trabajador, incentivando las jornadas a tiempo parcial. Adicionalmente, la extensión de las medidas que favorecen el cuidado de los niños.

\section{EFECTOS EN LA RELACIÓN DE TRABAJO Y RETOS PARA EL DERECHO SOCIAL}

La estrategia de flexiseguridad, tal como se ha podido desarrollar, ha tenido un efecto importante en la reconfiguración de los mecanismos de protección de la relación laboral, tanto en su constitución (modalidades de contratación temporal) como en la facilitación de su extinción.

Esta opción de política de empleo fomentada desde las instituciones europeas tiene como presupuesto la culpabilización del Derecho del Trabajo como causante de la pérdida de puestos de trabajo, lo cual desde una interpretación en contrario conduce a pensar que una menor protección del trabajador en la relación laboral y frente al empleador constituye un adecuado mecanismo de generación de empleo.

La experiencia europea no permite concluir que la flexibilización de las relaciones de trabajo haya generado más empleo. Lo que sí resulta claro es que ha conducido en la mayoría de los casos a una precarización de la contratación laboral, más aún si no existe el contrapeso del fomento de la regulación por la vía de la negociación colectiva.

El planteamiento de acudir a los mecanismos de protección social (principalmente al seguro de desempleo) como compensación por la facilitación del despido, salvo en los casos puntuales de Dinamarca y Países Bajos, no parece constituir un adecuado sustituto protector, tanto por su insuficiencia como por su extemporaneidad.

En un sentido similar, Joaquín Pérez Rey ha destacado que, en la práctica, la estrategia de flexiseguridad ha conducido al desequilibrio y extemporaneidad entre flexibilidad y seguridad. Así, señala que es difícil considerar que la asimetría contractual y el reparto de poderes en la empresa puedan ser equilibrados sobre la base de los sistemas de protección social.

Pérez Rey señala que tampoco debe soslayarse que el planteamiento de la flexiseguridad adolece de cierto maniqueísmo, condicionando el 
avance en la protección social a la flexibilización de la relación laboral, cuando lo cierto es que «avanzar en las políticas activas de empleo y en la adecuada protección de los desocupados es algo perfectamente compatible con el mantenimiento de unas relaciones laborales tuitivas y protectoras de la estabilidad $»^{35}$.

Además, debe tenerse en cuenta que la protección frente al cese en el puesto de trabajo no puede ser vista únicamente desde una perspectiva patrimonialista (protección en los ingresos) sino que debe considerar la importancia de la protección del trabajo como factor de inclusión y ciudadanía social.

Como lo han destacado Baylos Grau y Pérez Rey, siguiendo a Romagnoli, el «despido se debe encuadrar en la crítica del autoritarismo del poder privado en los lugares de trabajo y en la negación que conlleva del principio de igualdad de todos los ciudadanos ${ }^{36}$.

La consideración del trabajo como derecho fundamental y el respecto de la dignidad de la persona humana deben conformar límites infranqueables a la actuación arbitraria de los empleadores. La búsqueda de la igualdad sustancial como valor democrático sustenta esta opción protectora del trabajo.

Como ha señalado Baylos Grau, el planteamiento de la flexiseguridad conlleva la idea implícita de que «la mayor riqueza e intercambio mercantil requiere menos tutela de los derechos de los trabajadores. Esta era la música monocorde de los años noventa del pasado siglo» ${ }^{37}$. Destaca que no debe de perderse de vista que el Derecho del Trabajo constituye un nexo imprescindible entre la esfera de lo político-social y el ámbito de las relaciones económico-sociales. Se trata de una construcción política y democrática que relaciona dos grandes campos de la realidad, el de la economía de libre mercado y el de la política que determina en lo social las nociones clave de igualdad y solidaridad.

En este sentido, la que denomina «Constitución del trabajo» o «Constitución social» se define no solo por la delimitación de un perímetro cada vez más extenso de desmercantilización de las necesidades sociales, sino también por la asignación constitucional de valor político al trabajo, que en consecuencia impregna la actuación normativa y de gestión de los poderes públicos y de los sujetos sociales. 
En «Mercado y Sistema Jurídico Laboral en el nuevo siglo», Baylos Grau ${ }^{38}$ señala que la readecuación entre economía y ordenamiento laboral hace necesario desarrollar desde el Derecho Social, para que cumpla con sus objetivos de equidad y respeto de la dignidad de la persona humana, entre otros, los siguientes aspectos:

- Establecer nuevas condiciones de ejercicio del poder empresarial o procedimentalizar este poder

- Diseñar políticas de igualdad en las condiciones de empleo y la seguridad social

- Normalización del dialogo social y la negociación colectiva

- Reconocer dentro de la empresa la existencia garantizada de derechos fundamentales de los trabajadores en cuanto ciudadanos

- Creación de un piso mínimo de condiciones de vida y trabajo, con una regulación necesaria de la salud laboral, el salario, el tiempo de trabajo y de vida

Estos retos, si bien han sido elaborados para la realidad europea, también se plantean como tales para el futuro del Derecho del Trabajo en Latinoamérica. Y ello es así, en buena cuenta, porque a pesar de las diferencias económicas, sociales y culturales que separan a Europa de los países latinoamericanos, la misión histórica asignada al Derecho del Trabajo en la mayoría de constituciones latinoamericanas se sustenta en un conjunto de principios y valores comunes a la cultura occidental moderna. A saber, el respeto de la dignidad de la persona humana, el valor del trabajo en la construcción de ciudadanía social y el impulso de «lo colectivo» como mecanismo de democratización.

\section{ALGUNAS LECCIONES PARA EL SUR}

Desde inicios de la década de 1990, en varios países latinoamericanos se implementaron reformas dirigidas a flexibilizar las relaciones laborales. En el caso peruano, como en algunos otros, estas reformas no fueron producto de la participación de los interlocutores sociales sino que, por el contrario, fueron impuestas e inclusive se sustentaron en medidas que buscaban limitar (sino eliminar) la actuación sindical en las empresas y la vida nacional ${ }^{39}$.

38 Baylos Grau, Antonio. «Mercado y Sistema Jurídico Laboral en el Nuevo Siglo (Notas para debatir con Moisés Meik)". Notas del autor sobre la base de materiales anteriores empleados en Brasil y México en el 2005. Materiales de lectura del Máster de Empleo, Relaciones Laborales y Diálogo Social en Europa. Universidad de Castilla-La Mancha, 2010.

$39 \mathrm{En}$ realidad, el primer antecedente en estas reformas desreguladoras fue la implementada en Chile en 1979 durante la dictadura de Augusto Pinochet. En ese caso, se trató del llamado Plan Laboral, que facilitó las formas de contratación temporal y el despido. Quien fuera ministro de Trabajo y Previsión Social en aquella época relata descarnadamente que la «tarea número uno fue, necesariamente, parar el boicot del sindicalismo internacional». Un segundo paso sería la reforma

EL DEBATE

EUROPEO SOBRE

LA FLEXI-

SEGURIDAD:

ALGUNAS

LECCIONES PARA

EL SUR

THE EUROPEAN

DEBATE ON

FLEXISECURITY:

SOME LESSONS

FOR THE SOUTH 
La reforma laboral peruana se dio en el contexto de una reforma estructural que tuvo como objetivos la liberalización de la economía, una apertura comercial exterior sin precedentes y la privatización de la actividad empresarial del Estado. Adicionalmente, en un contexto de crisis económica y altas tasas de inflación, la reforma laboral y de la previsión social fue anunciada como una dimensión más de las medidas que buscaban otorgar mayor estabilidad económica al país ${ }^{40}$.

La flexibilización laboral peruana, calificada como de «desrregulación salvaje» ${ }^{41}$, no varió significativamente hasta el año 2001. Sin embargo, las pocas modificaciones al régimen laboral han estado vinculadas con la reforma de las relaciones colectivas del trabajo (nivel de negociación) y la jurisprudencia del Tribunal Constitucional (reposición en casos de despidos nulo, fraudulento o sin causa). En los años recientes, se han creado «regímenes especiales» para los trabajadores del Estado (denominados de Contratación Administrativa de Servicios) y de las micro empresas, los cuales tienen menores condiciones de empleo y protección social que el régimen general.

Un aspecto que conviene destacar, como señalamos al inicio de este texto, es que la reforma laboral iniciada el año 1991 se sustentó en una política de generación de empleo. Así, la norma laboral más importante tenía como denominación «Ley de Fomento del Empleo». El problema desde entonces en el Perú, como ahora, no era fundamentalmente la falta de empleo sino la baja calidad de este. Por lo que se requiere, antes como ahora, de políticas que fomenten el trabajo decente y una mayor productividad.

Es decir, la reforma laboral peruana, se sustentó, según Baylos Grau, en la música monocorde de los años ochenta (incluso ya pasada de moda en 1991) que buscaba culpar al Derecho Laboral de la pérdida de puestos de trabajo. Con el agravante de que en el Perú la subordinación de la protección del trabajo al fomento del empleo no tuvo como fundamento la realidad del mercado de trabajo peruano sino un discurso ideológico que buscaba ser hegemónico y fue impuesto.

Los lineamientos de esta reforma, en lo que respecta a la flexibilidad externa de entrada, se mantienen hasta la actualidad, siendo así que en el Perú la mayoría de trabajadores asalariados son contratados de manera temporal, superando largamente la tasa técnica de temporalidad,

previsional sustentada con la implementación de un sistema de capitalización individual. Ver PIÑERA, José. El cascabel al gato. La batalla por la reforma previsional. Santiago: Zig-Zag, 1991, p. 16.

40 Una manifestación de ello fueron los compromisos que asumía el Estado Peruano frente a organismos financieros multilaterales como el Banco Mundial y el Fondo Monetario Internacional (Cartas de Intención), en los que se obligaba a implementar una reforma que flexibilice la legislación laboral y privatice el ámbito de la previsión social.

41 ERMIDA URIARTE, Oscar. «La flexibilidad». En AUtORES VARIOS. Estudios sobre la flexibilidad en el Perú. Lima: Organización Internacional del Trabajo/Oficina de Área y Equipo Técnico Multidisciplinario para los Países Andinos, 2000. 
con el efecto pernicioso que ello tiene en la productividad del trabajo. Asimismo, se permite la intermediación laboral (provisión de personal) no solamente mediante empresas de trabajo temporal, sino también para actividades denominadas complementarias o altamente especializadas.

A ello habría que agregar que más de un tercio del empleo peruano es calificado como autoempleo ${ }^{42}$. Es decir, personas que no prestan servicios de manera dependiente, y en consecuencia no cuentan con protección laboral ni social de calidad, debido al modelo contributivo de seguridad social existente en el Perú. Modelo reforzado por un discurso ideológico predominante en el espacio público, por el cual, con el argumento de premiar la iniciativa privada, se hace a cada uno responsable de su suerte.

Más aún, la flexibilización externa de salida no fue acompañada de mecanismos de protección social implementados desde el Estado, ya que en el Perú no existe un seguro de desempleo, sino un beneficio de cesantía sustentado en el ahorro individual privado.

La cobertura poblacional de la seguridad social en el Perú es en salud de alrededor del $36 \%$ y de $22 \%$ en pensiones, existiendo una gran mayoría privada de acceso a protección social ${ }^{43}$. Los programas de prestaciones familiares son prácticamente inexistentes y se concentran en programas de lucha contra la pobreza de manera focalizada.

Respecto del empleo, tan solo el 43\% de las mujeres de Lima Metropolitana se encuentran adecuadamente empleadas, mientras que en el caso de los varones esta cifra asciende al $56 \%$, datos que tienden a agravarse en el interior del país. Esto se condice con el hecho de que, si bien en los últimos años el empleo creció, el aumento se concentró en el subempleo. A su vez, el incremento de la demanda de mano de obra calificada ha acentuado el diferencial de ingresos y las desigualdades.

En buena cuenta, la flexibilidad laboral en el Perú no fue acompañada de seguridad social sino de indefensión, generando mayor inequidad y desprotección social, produciendo un modelo que podríamos denominar, sin exageraciones, de «flexi-inseguridad».

A partir del debate europeo sobre la flexiseguridad y los resultados de dos décadas de reforma laboral peruana, consideramos importante extraer algunas conclusiones para una futura reforma protectora del Derecho Social en el Perú:

42 En términos de Romagnoli, «Se ha erosionado la presunción favorable a la subordinación: el trabajo declina en plural y el autónomo está incrementado su dimensión. Por doquier» (ROMAGNOLI, Umberto. «¿Un arca de Noé para el Derecho del Trabajo?». Bolonia, 15 de septiembre de 2007. Lección inaugural Curso OIT - Universidad de Bologna-Univesidad de Castilla - La Mancha.

43 VIDAL, Álvaro. «El Estado de Bienestar en el Perú: La distancia entre lo real y lo imaginario». En VILLAVICENCIO, Alfredo (coord.). Temas Centrales del Derecho del Trabajo del S. XXI. Lima: Ara Editores, 2009. 
- La necesidad de una relación de equilibrio entre políticas de empleo y políticas sociolaborales que tengan en cuenta la realidad del mercado de trabajo y sus tendencias pasadas y futuras. Promover el crecimiento económico superando visiones ideologizadas que culpan al Derecho del Trabajo de la crisis del empleo.

- Evaluar el resultado de la extensión mayoritaria de la contratación temporal y su efecto en la productividad. A partir de ello, regular adecuadamente la flexibilidad externa de entrada, mediante causales objetivas y no subjetivas, (superar el errado argumento de fomento de la contratación de «franjas débiles»).

- Reconocer el valor del trabajo decente como herramienta para la inclusión social y construcción de ciudadanía, al interior y fuera de la empresa. Por ello, la importancia de regular el ejercicio del poder privado dentro de la organización productiva como ejercicio democrático (regulación protectora del trabajo al interior de la relación laboral).

- Fomentar la organización sindical y su función no solamente reguladora sino como sujeto político e interlocutor necesario en el diseño e implementación de políticas sociales.

- Desarrollar mecanismos de compensación de la flexibilidad laboral mediante sistemas de protección social. En particular, el establecimiento de mínimos de protección social en materia de desempleo, salud, pensiones y educación.

- Fomentar la capacitación permanente y la innovación como formas de generar mayor productividad. Establecer políticas activas de empleo y protección especial para los mercados transicionales (transición de empleos, entre formación y empleo, desempleo y empleo, actividades no comerciales y empleo, y entre empleo e inactividad). 